magnet the egg-shaped luminosity of greenish-yellow colour which is formed between the electrodes in a discharge-tube of wider diameter containing pure oxygen at low pressure. At the points where the deflected egg is brought near to the wall, two patches of brilliant red appear, separated by narrow, dark inter-spaces from the rim of gold-coloured fluorescence next to the electrodes. With other gases quartz fluoresces in the deep blue or violet, sometimes in the green, whereas the red fluorescence, corresponding to a band near $620 \mu \mu$, is only observed with oxygen at low pressure. Hans Pettersson.

\section{Structure of the Band Spectra of the Hydrogen and Helium Molecules.}

IN the spectrum of the hydrogen molecule many regularities have been found recently, especially by Richardson and his co-workers. In a note in the $Z s$. f. Physik I suggested an interpretation of those regularities based mainly on the theory of band complexes and the analogy with the helium band spectrum. The analogy was incomplete in so far as the bands found in the spectrum of the hydrogen molecules are analogous to helium bands which can be predicted from theoretical considerations, but which had not been actually found. I have found these missing helium bands now. Their structure is exactly analogous to that of the hydrogen bands given by Richardson and Davidson (Proc. Roy. Soc., A, 123, 54, 466, A, 124, 50, 69), as will be best apparent from a descrintion of their peculiarities. From the red to the violet we have the following branches:

\begin{tabular}{|c|c|c|c|}
\hline Transition. & $\begin{array}{c}\text { Description of the } \\
\text { Bands. }\end{array}$ & Richardson's. & $\begin{array}{l}\text { Finkelburg } \\
\text { and Mecke. }\end{array}$ \\
\hline$\sigma \mathbf{\Sigma} \longrightarrow 2 \pi \Sigma$ & $P$. and $R$-branch of & ${ }^{1} K \longrightarrow 2^{1} S$ & ${ }^{8} D \longrightarrow 2^{3} S$ \\
\hline $\begin{array}{l}\delta \Sigma \longrightarrow 2 \pi \Sigma \\
\delta \Pi_{b} \longrightarrow 2 \pi \Sigma \\
\delta \Pi_{a \rightarrow 2 \pi \Sigma} \rightarrow 2\end{array}$ & $\begin{array}{l}R \text {-strong, } P \text {-weak. } \\
\text { Only strong } Q \text {. } \\
P \text { strong, } R \text { weak. }\end{array}$ & $\begin{array}{l}{ }^{1} \mathrm{C} \rightarrow{ }^{2} \mathrm{~S} \\
{ }^{1} \mathrm{~B} \rightarrow 2^{1} S \\
{ }^{1} \mathrm{~A} \rightarrow 2^{1} S\end{array}$ & $\begin{array}{l}-2^{3} S \\
-2^{3} S \\
-2^{3} S\end{array}$ \\
\hline
\end{tabular}

In addition to these seven branches there is one more $P-, Q$-, and $R$-branch arising from $\delta \Delta_{a, b} \rightarrow 2 \pi \Sigma$ transitions. These branches are very faint, and their intensities make it probable that they are only present if the regular precession of the orbital electronic moment of momentum around the nuclear axis is considerably perturbed. It is not yet quite certain to which hydrogen bands these three branches correspond. If one takes these facts together with the arguments mentioned in the note in the Zs.f. Phys., the evidence in favour of the proposed explanation of the hydrogen bands becomes very strong. The properties of the helium terms are well known (see the letter to NATuRE of May 11 and a fuller discussion in print in Zs.f. Phys.), and therefore I think there is no reason to accept the conception of Finkelburg and Mecke (Zs. f. Phys., 54, p. 537) of the hydrogen bands which is given in the last column of the table.

All the bands the analysis of which seems most certain find their explanation in this way. The interpretation of some of the remaining terms does not seem to be easy. There are reasons, however, which make it not improbable that the assignment of initial vibrational and electronic quantum numbers ought to be changed for some bands. In such cases Richardson and Davidson's and Finkelburg and Mecke's analyses usually do not agree with each other.

A few words may be added about the newly discovered helium bands. There are three groups of them, all belonging to the triplet system, one in the red $(4 \sigma$ and $4 \delta \rightarrow 2 \pi \Sigma)$, one near $535 m \mu$, and one near $495 \mathrm{m \mu}$ (5\% and $5 \delta$ resp. $6 \sigma$ and $6 \delta \rightarrow 2 \pi \Sigma)$.

No. 3113 , VoL. 123]
The group near 535 was first found by Merton and Pilley. It and the 495 group have been partly analysed by Fujioka ( $Z s$. f. Phys., 52, p. 657). All the bands are degraded toward the violet. The initial terms were known from other bands, the new final term $2 \pi \Sigma$ lies $6118.4 \mathrm{~cm}^{-1}$ above the corresponding $2 \pi \Pi$ - $(2 p$-) term, whereas in hydrogen the $2 \pi \Sigma$-level ( $B$-level) lies $8892 \mathrm{~cm}^{-1}$ below the $2 \pi \mathrm{II}$ - $(C \cdot)$ level. This is the most remarkable difference between the hydrogen and the helium terms, whereas in most other respects they are exactly analogous. That will be seen more clearly from the detailed paper. The new helium bands will be described in collaboration with Messrs. Takamine and Imanishi. Their discovery also made possible the hitherto doubtful analysis of bands in the region around $400 \mathrm{~m} \mu$ and $378 \mathrm{m \mu}$. It appears that perturbations of the kind described in my letter to NATURE of Mar. 23 occur for the $\sigma \Sigma-(s-)$ and $\delta \Sigma-(z-)$ terms. The perturbation moves to lower $j$ if we go to the higher terms. $4 z(17), 5 z(9)$, and $6 z(5)$ are the perturbed $z$-terms.

Natuurkundig Laboratorium

G. H. Dreke. der Rijks-Universiteit, Groningen.

\section{The Primary Process in the Formation of the Latent Photographic Image.}

I HAVE read with much interest the communication from Dr. F. C. Toy and Mr. G. B. Harrison in Nature of May 4, 1929, p. 679. The experiments described on the photo-conductance phenomenon in silver bromide afford valuable confirmation of the results obtained by Dr. W. Vanselow and myself on the photo-voltaic effect at silver bromide : silver electrodes, which were briefly described in the sixth Hurter and Driffield Memorial Lecture. ${ }^{1}$ These results, we consider, not only demonstrated the primary separation of electrons by light in the photolysis of silver bromide, but also gave the first evidence that this separation of electrons is actually related to the liberation of bromine. The negative potential difference ascribed to electron liberation is produced within $\frac{1}{6} \sigma$ second of the incidence of the light, attaining a maximum within $\frac{1}{65}$ to $\frac{1}{2} 0$ second.

We regard these, and other results now being published in the Journal of Physical Chemistry, as confirming the hypothesis of electron liberation from the bromide ion and transfer to the silver ion, which was proposed by Sheppard and Trivelli, and independently by Fajans, in 1921. Dr. Toy and Mr. Harrison interpret their recent results in terms of this same theory. Now it may be noted that the photo. conductance phenomenon by itself only shows the production of mobile electrons, but not that they are valence electrons from the bromide ions. The correspondence of the primary photo-conductance current with the photographic effect, as demonstrated for wave-length sensitivity and time-order sensitivity by Dr. Toy and his collaborators, is unquestionably very significant. Considered in relation with our measure. ments of the photo-voltaic effect, they strongly support the view that the inner photo-electric effectsphoto-voltaic and photo-conductance-as also the photographic and photochemical effects, all derive from the same primary separation of the electron from the bromide ion.

In terming this the primary event, I take leave to differ somewhat from Dr. Toy and Mr. Harrison in regard to their statement on latent image formation.

1 "The Formation of the Photographic Latent Image", Phot. J., 67, $397-414 ; 1928$ 